\title{
Neuropsychiatric systemic lupus erythematosus in a geriatric patient: A case report
}

\author{
Shalini Sri Kumaran*1, Annie Law Hui $\mathrm{Nee}^{2}$ \\ ${ }^{1}$ Department of Internal Medicine, Singapore General Hospital, Singapore \\ ${ }^{2}$ Department of Rheumatology \& Immunology, Singapore General Hospital, Singapore
}

Received: October 23, 2016

DOI: $10.5430 /$ crim.v4n1p50
Accepted: January 6, 2017

Online Published: January 13, 2017

\begin{abstract}
Systemic lupus erythematosus (SLE) is a multisystem connective tissue disorder with varied clinical presentations with late onset SLE occurring after the age of 50. Involvement of the central nervous system (CNS) in SLE may range from non-focal symptoms of cognitive dysfunction, an acute confusional state, to focal symptoms of transient ischaemic attacks and strokes. We report a case of an elderly male who presented with confusion, functional decline and fevers. On admission, he was febrile with a Glasgow Coma Scale (GCS) of 11 with no other focal neurological or systemic examination findings. Initial investigations pointed towards a diagnosis of possible tuberculous meningitis with a differential diagnosis of unidentified sepsis and malignancy still being entertained. However, a newly developed purpuric skin rash helped clinch the diagnosis of SLE and he was finally diagnosed as a case of late-onset neuropsychiatric systemic lupus erythematosus (NPSLE). He was started on intravenous (IV) hydrocortisone which was later switched to a tapering dose of oral prednisolone and made a significant improvement.
\end{abstract}

Key Words: Confusion, Elderly, Late-onset systemic lupus erythematosus, Neuropsychiatric systemic lupus erythematosus

\section{INTRODUCTION}

Systemic lupus erythematosus (SLE) is a multisystem autoimmune disorder with a wide range of clinical and paraclinical involvement that also has a variable course of prognosis and response to treatment. It is typically known to affect women at their reproductive age. However, with higher life expectancy in the past decades, "late-onset SLE", defined by diagnosis at or after the age 50 , have been reported to occur in 3\%-20\% of patients. ${ }^{[1,2]}$ Late-onset SLE has been widely discussed as their disease course, manifestations and prognosis differs from patients with younger onset.

Cohort studies have shown that late-onset SLE are more likely to have an insidious presentation with more nonspecific symptoms such as myalgias, fatigue, weight loss, neurological involvement, and less renal involvement when compared to younger onset SLE. ${ }^{[3-5]}$

We report a case of an elderly Asian man who presented as the typical geriatric patient who was later diagnosed with late-onset SLE.

\section{Case report}

A 73-year-old Chinese male was admitted to an acute tertiary hospital in July 2014, after a fall, with a 1-month history of functional decline and cognitive impairment. He was found by his family to get gradually weaker with the patient needing some assistance in the initial stages to full assistance with all his daily care needs such as having a bath and even eating. A more detailed history was difficult to obtain given

*Correspondence: Shalini Sri Kumaran; Email: sri.kumaran.shalini@singhealth.com.sg; Address: Department of Internal Medicine, Academia Level 4, 20 College Road, Singapore. 
his confusional state but the family clarified that they noticed him becoming less focused and forgetting things in the month prior to admission with instances where the patient would forget where he was or would not recognize his family members. They described him as almost "wasting away" with noticeable weight loss though unable to be quantified and disorientation with physical weakness to the point of needing a full carer.

He has a past history of hypertension and was on amlodipine (7.5 mg daily). He had no known dementia nor psychiatric history. He did not consume alcohol nor tobacco.

On examination, he was febrile with a temperature of $39.2^{\circ} \mathrm{C}$, tachycardic with a pulse rate of $116 \mathrm{bpm}$, blood pressure of 170/70 $\mathrm{mmHg}$, alert but confused with a Glasgow Coma Scale (GCS) of 11 points. Neurologically, he had some mild diffuse muscle weakness with a power of 4+ throughout all his limbs with no focal abnormalities, and there were no signs of meningism. He could obey simple one step commands but could not concentrate to hold a conversation and answer questions accurately. Cardiovascular, respiratory and abdominal examination were unremarkable. There were no palpable lymphadenopathy nor joint effusions or erythema detected.

Initial investigations revealed pancytopaenia with a haemoglobin $(\mathrm{Hb})$ of $7.6 \mathrm{~g} / \mathrm{dl}$, white blood count (WBC) of $3.74 \times 10^{9} / \mathrm{L}$, and a platelet count of $74 \times 10^{9} / \mathrm{L}$, elevated inflammatory markers with a C-reactive protein (CRP) of $142 \mathrm{mg} / \mathrm{L}$, procalcitonin of $3.5 \mu \mathrm{g} / \mathrm{L}$, and an erythrocyte sedimentation rate (ESR) of $106 \mathrm{~mm} / \mathrm{HR}$. Acute kidney injury (AKI) with a raised creatinine of $165 \mathrm{ummol} / \mathrm{L}$ and a urea level $8.9 \mathrm{mmol} / \mathrm{L}$, and mildly low albumin of $31 \mathrm{~g} / \mathrm{L}$. His urinalysis revealed pyuria of $>2,000$ white cells and the urine culture grew Enterococcus faecalis. He was therefore started on IV ceftriaxone. His condition did not improve despite day five of antibiotics and he continued to have persistent fever.

A computed tomography (CT) of the thorax, abdomen and pelvis revealed significant lymphadenopathy and a CT brain showed chronic microvascular ischaemic changes. HIV serology was negative as was a transthoracic echocardiogram. A lumbar puncture was done and showed elevated protein (1.63 g/L, normal: $0.1-04 \mathrm{~g} / \mathrm{L})$ with only $1 \mathrm{WBC}$ cell seen, and no evidence of malignant cells. An excisional right iliac fossa lymph node biopsy was done and it was also negative for malignancy. Hence, a presumptive diagnosis of tuberculous meningitis and lymphadenitis was made and the patient was started on empirical anti-TB medication while awaiting final results.

However, despite anti-TB drugs, he continued to have fluctu- ating levels of consciousness with intermittent fever of $39^{\circ} \mathrm{C}$. TB cultures and TB DNA amplification assay eventually resulted negative as well. A week on, a new scaly purpuric rash had developed over the patient's forearms which worsened when he was seated by the window in his room (see Figure 1). A punch biopsy of the rash revealed interface dermatitis with confluent vacuolar degeneration and scattered cytoid bodies, features consistent with lupus erythematosus.

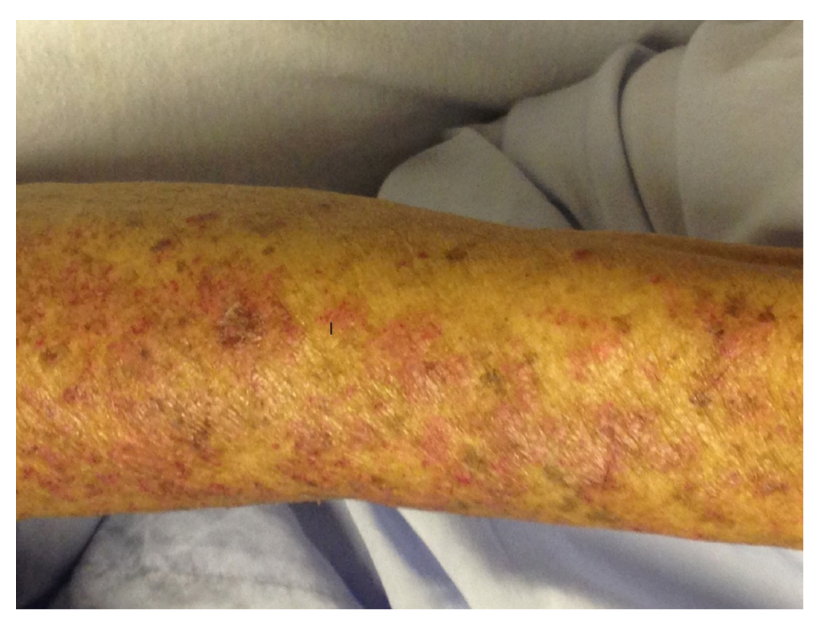

Figure 1. Photosensitive systemic lupus erythematosus (SLE) rash that appeared acutely on the patient's forearm

This prompted us to further evaluate for SLE and the results were a positive antinuclear antibody (ANA) of $>1: 800$ titre (homogenous pattern), a positive anti double-stranded DNA (anti ds-DNA) of $334 \mathrm{IU}$ (normal is < $25 \mathrm{IU}$ ), hypocomplementaemia: low C3 0.39 (normal $0.45-0.83 \mathrm{G} / \mathrm{L}$ ) and low C4 $0.06 \mathrm{G} / \mathrm{L}$ (normal $0.11-0.41 \mathrm{G} / \mathrm{L}$ ), and the presence of proteinuria $1.62 \mathrm{~g}$ with no urinary casts seen. The patient was negative for other extractable nuclear antigens such as anti-Ro, anti-La and anti-Smith.

On the basis of the positive serology, hypocomplementaemia, pancytopaenia, proteinuria, photosensitive rash and his acute confusional state likely being a neuropsychiatric (NP) manifestation a diagnosis of possible NPSLE was made. He remained on the anti-TB drugs and was started on IV hydrocortisone once the cerebrospinal fluid cultures and acid fast bacilli (AFB) smears were negative. His mental state improved significantly and he was able to participate in rehabilitation. He was back at his baseline mental function when reviewed in the outpatient clinic whilst on a tapering dose of prednisolone. His anti-TB drugs were discontinued once the final TB cultures were negative.

\section{Discussion}

SLE is an autoimmune disorder with variable clinical and immunological expressions that requires a high index of sus- 
picion for its diagnosis especially in the elderly where it can be the great mimicker of many other more prevalent conditions. Given this heterogeneity, the American College of Rheumatology (ACR) first came up with criteria to aid in the diagnosis of SLE in 1982 which were then revised in 1997. Since then, a few pitfalls were noted such as the omission of hypocomplementaemia which has been shown to be one of the single most powerful criteria for $\mathrm{SLE}^{[6]}$ and thus the Systemic Lupus International Collaborating Clinics (SLICC) Classification 2012 criteria were derived. The SLICC criteria has shown to retain the specificity of the original ACR criteria while being more sensitive. ${ }^{[6]}$

Studies done on late-onset SLE have reported conflicting data mainly because of limitations such as different age cut-offs that define late-onset, small sample size, variable ethnicity composition, and the different clinical and immunological data reported. A review done by Lazaro, ${ }^{[2]}$ which summarised the clinical features of late-onset SLE from 13 studies, reported that arthritis, fever, rash and serositis were the most common symptoms seen. However, another review by Arnaud et al. ${ }^{[1]}$ reported that arthritis and rash occurred less commonly.

Cutaneous manifestations such as malar, discoid and photosensitive rash, mucosal ulcerations, sicca symptoms, Raynaud's phenomenon and alopecia are common findings in SLE with them representing $50 \%$ of the SLICC clinical criteria. ${ }^{[6]}$ However, data literature within the late-onset group is conflicting. A very recent systematic review and metaanalysis done by Medlin et al. ${ }^{[7]}$ found that, while cutaneous signs are still common, they are less common compared to early-onset SLE with malar rash, photosensitivity and alopecia occurring less frequently and sicca symptoms being more common among the elderly group. This differed in our patient, in which the photosensitive rash helped us to clinch the diagnosis. The hypothesis behind fewer cutaneous manifestations in the elderly may be due to immune senescence. ${ }^{[8]}$

Hebra and Kaposi were the first to note CNS involvement in lupus back in 1875 and since then reports have shown that the neurological manifestations of SLE can precede the onset or occur at any time during the course of the disease. ${ }^{[9]}$ NPSLE includes the neurologic syndromes of the central, peripheral and autonomic nervous system in addition to the psychiatric syndromes seen in patients in which other causes have been excluded. ${ }^{[10]}$ Given the myriad of symptoms that it encompasses, an expert committee by the ACR took to the task of classifying NPSLE and, in 1999, they identified 19 neuropsychiatric conditions which included $12 \mathrm{CNS}$ and 7 peripheral nervous system.

A meta-analysis by Unterman et al. ${ }^{[11]}$ reported that the prevalence of NPSLE amongst all age groups was $56 \%$ with headache, mood disorders, and cognitive dysfunction being most frequently observed. However, there has been contradicting results concerning NPSLE in late-onset SLE patients with most studies reporting lower frequencies. ${ }^{[12,13]}$ This is not surprising as quite often a more common differential diagnosis is attributed to the symptom such as depression or an infective aetiology such as tuberculous meningitis as initially presumed to be in our patient. The pathogenesis of NPSLE is still poorly understood with several pathogenic pathways being linked to specific clinical manifestations such as antibody-mediated neurotoxicity, vasculopathy due to antiphospholipid antibodies, cytokine-induced neurotoxicity and loss of neuroplasticity ${ }^{[14]}$ with no test or imaging modality been found to contribute significantly to the work-up of NPSLE. Studies done using single proton emission computed tomography (SPECT) were thought to be more sensitive in evaluating NPSLE but results have been inconsistent. ${ }^{[1]}$

Late-onset SLE has been reported to present with less aggressive features such as lupus nephritis, ${ }^{[1]}$ however the prognosis and mortality appears to be high in this group. ${ }^{[2]}$ This is probably due to factors such as declining immune system in the elderly, comorbidities in addition to the burden of disease itself, and pharmacologic interactions with other drugs that the patient is already on. ${ }^{[1-3]}$

\section{Conclusion}

In conclusion, this case highlights the insidious nature of lateonset NPSLE and the great mimicker it can be. It also illustrates that disease presentations are frequently non-specific in the elderly, and many a times we are dealing with red herrings and looking beyond our usual differentials is needed.

\section{CONFLICTS OF INTEREST Disclosure}

The authors have no competing interests to declare.

\section{REFERENCES}

[1] Arnaud L, Mathian A, Boddaert J, et al. Late-onset Systemic Lupus Erythematosus Epidemiology, Diagnosis and Treatment. Drugs Aging. 2012; 29(3): 181-189. PMid:22263748 https ://doi.org/10
$.2165 / 11598550-000000000-00000$

[2] Lazaro D. Elderly-Onset Systemic Lupus Erythematosus Prevalence, Clinical Course and Treatment. Drugs Aging. 2007; 24(9): 701-715. PMid:17727302 https://doi.org/10.2165/00002512-20072 
4090-00001

[3] Alonso MD, Martinez-Vazquez F, Diaz de Teran T, et al. Lateonset systemic lupus erythematosus in Northwestern Spain: differences with early-onset systemic lupus erythematosus and literature review. Lupus. 2012; 21: 1135-1148. PMid:22652632 https : //doi.org/10.1177/0961203312450087

[4] Lin H, Wei JC, Tan CY, et al. Survival analysis of late-onset systemic lupus erythematosus: A cohort study in China. Clin Rheumatol. 2012; 31: 1683-1689. PMid:22983769 https ://doi.org/10.1007/s1 0067-012-2073-6

[5] Tomic-Lucic A, Petrovic R, Radak-Perovic M, et al. Late-onset systemic lupus erythematosus: Clinical features, course and prognosis. Clin Rheumatol. 2013; 32: 1053-1058. PMid:23515605 https://doi.org/10.1007/s10067-013-2238-y

[6] Shankar S, Pathak A. Redefining Lupus in 2012 [Internet]. Chapter. 99: 449-51. Available from: http://www.apiindia.org/medic ine_update_2013/chap99.pdf

[7] Medlin JL, Hansen KE, Fitz SR, et al. A Systematic Review and Meta-Analysis of Cutaneous Manifestations in Late Versus EarlyOnset Systemic Lupus Erythematosus. To appear in: Seminars in Arthritis and Rheum. https://doi.org/10.1016/j.semarthr it. 2016.01.004

[8] Hashimoto H, Tsuda H, Hirano T, et al. Differences in clinical and immunological findings of systemic lupus erythematosus related to age. J Rheumatol. 1987; 14(3): 497-501. PMid:3625631

[9] Fady JG, Lammie AG, Scolding NJ. CNS lupus A study of 41 patients. Neurology. 2007; 69: 644-654. PMid:17698785 https : //doi.org/10.1212/01.wnl.0000267320.48939.d0

[10] The American College of Rheumatology nomenclature and case definitions for neuropsychiatric lupus syndromes. Arthritis Rheum. 1999; 42: 599-608. https://doi.org/10.1002/1529-0131(199904 ) $42: 4<599:$ :AID-ANR2>3.0.CO;2-F

[11] Unterman A, Nolte JE, Boaz M, et al. Neuropsychiatric syndromes in systemic lupus erythematosus: a meta-analysis. Semin Arthiritis Rheum. 2011; 41: 1-11. PMid:20965549 https ://doi.org/10.1 016/j.semarthrit.2010.08.001

[12] Boddaert J, Huong DL, Amoura Z, et al. Late-onset systemic lupus erythematosus: a personal series of 47 patients and pooled analysis of 714 cases in literature. Medicine (Baltimore). 2004; 83: 348-359. https://doi.org/10.1097/01.md.0000147737.57861.7c

[13] Lalani S, Pope J, de Leon F, et al. Clinical features and prognosis of late-onset systemic lupus erythematosus: Results from the 1000 Faces of Lupus Study. J Rheumatol. 2010; 37(1): 38-44. PMid:20008925 https://doi.org/10.3899/jrheum.080957

[14] Kivity S, Agmon-Levin N, Zandman-Goddard G, et al. Neuropsychiatric lupus: a mosaic of clinical presentations. BMC Medicine. 2015; 13: 43. PMid:25858312 https://doi .org/10.1186/s12916-0 15-0269-8 